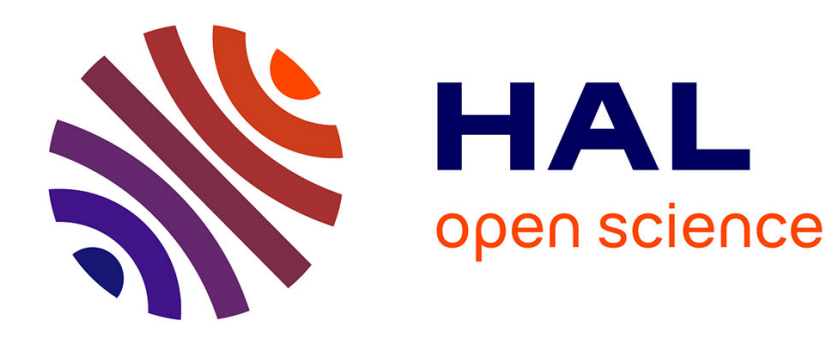

\title{
La flexibilité du travail en Corée du Sud
}

Bruno Jetin

\section{To cite this version:}

Bruno Jetin. La flexibilité du travail en Corée du Sud. Revue Tiers Monde, 1998, 39 (154), pp.403-423. 10.3406/tiers.1998.5246 . halshs-02043383

\section{HAL Id: halshs-02043383 \\ https://shs.hal.science/halshs-02043383}

Submitted on 21 Feb 2019

HAL is a multi-disciplinary open access archive for the deposit and dissemination of scientific research documents, whether they are published or not. The documents may come from teaching and research institutions in France or abroad, or from public or private research centers.
L'archive ouverte pluridisciplinaire HAL, est destinée au dépôt et à la diffusion de documents scientifiques de niveau recherche, publiés ou non, émanant des établissements d'enseignement et de recherche français ou étrangers, des laboratoires publics ou privés. 


\section{Bruno Jetin}

\section{La flexibilité du travail en Corée du Sud}

In: Tiers-Monde. 1998, tome 39 n¹54. pp. 403-423.

Citer ce document / Cite this document :

Jetin Bruno. La flexibilité du travail en Corée du Sud. In: Tiers-Monde. 1998, tome 39 n¹54. pp. 403-423.

doi : 10.3406/tiers.1998.5246

http://www.persee.fr/web/revues/home/prescript/article/tiers_1293-8882_1998_num_39_154_5246 


\title{
LA FLEXIBILITÉ DU TRAVAIL EN CORÉE DU SUD
}

\author{
par Bruno JETIN*
}

L'objectif de cet article est de montrer que la flexibilité quantitative et la flexibilité fonctionnelle du travail, loin de s'opposer, sont au contraire complémentaires. Une flexibilité quantitative élevée est une condition nécessaire au développement de la flexibilité fonctionnelle à un coût compatible avec les exigences de la compétitivité. Telle pourrait être une des conséquences de la crise qu'a connue la Corée à la fin de 1997.

Depuis l'entrée en crise des pays développés et l'émergence d'un chômage de masse, la flexibilité du travail est devenue un objet permanent du débat économique. Identifiée au libre fonctionnement du marché du travail, elle serait une condition du retour au plein-emploi en autorisant une baisse du coût du travail. En Corée, le débat est plus récent. Il est apparu avec l'adhésion à l'OCDE fin 1996, et s'est renforcé au début de l'année 1997, à l'occasion de la protestation sociale qui a suivi le vote d'une loi visant à développer la flexibilité du travail' ${ }^{1}$. Ce décalage tient à ce qu'en Corée, pendant longtemps, la flexibilité du travail était censée résoudre un tout autre problème. Non pas le retour au plein-emploi, car il existait déjà, mais la pénurie de travail, conséquence d'un taux de croissance élevé. Ce constat rappelle, s'il en était besoin, combien la flexibilité du travail, une des caractéristiques du rapport salarial, est

\footnotetext{
* Maitre de conférences à l'Université de Rouen. membre du CARE, Université de Rouen el du GREITD. Université Paris 13. Nous tenons à remercier Hwan Joo Seo pour son aide dans la réflexion et dans la documentation. Il n'en demeure pas moins que les idées exprimées sont de la seule responsabilité de l'auteur.

1. Cette loi, votée en catimini à la fin de l'année 1996, visait à supprimer les obstacles aux licenciements, à proroger l'existence d'une seule centrale syndicale. et à renforcer les prèrogatives des services de renseignements dans le domaine social. Après avoir été modifiée. son entrée en vigueur a été reportée de deux ans.
} 
ambiguë et protéiforme'. Parmi les nombreuses taxinomies proposées, on retiendra celle de l'OCDE ${ }^{2}$ qui distingue la flexibilité quantitative de la flexibilité fonctionnelle. La première désigne la possibilité d'adapter le volume de l'emploi et des heures travaillées aux variations de l'activité des entreprises au moyen des licenciements et de l'externalisation d'une partie de la production. La seconde, de nature plus qualitative, concerne la capacité de l'organisation de la production et du travail, ainsi que des relations sociales au sein des entreprises, à s'adapter aux variations du volume et de la composition de la demande. Ces deux types de flexibilité satisfont le même objectif, améliorer la réactivité des entreprises, mais ne peuvent être comprises que si on les met en rapport. Plus précisément, la flexibilité quantitative se distingue par le fait qu'elle fait pénétrer la «discipline» du marché du travail au sein de l'entreprise. Ainsi, plus le chômage sera élevé, plus la pression à la baisse qui s'exercera sur les salaires et les conditions d'emploi au sein de l'entreprise sera forte. C'est pourquoi notre hypothèse de travail est que la flexibilité quantitative détermine à quel coût et selon quelles modalités la flexibilité fonctionnelle pourra se développer au sein des entreprises. Dans ces conditions l'analyse de ces deux types de flexibilité du travail ne peut faire abstraction des conditions historiques qui caractérisent le régime d'accumulation en vigueur.

Cet article commencera donc (section 1) par rappeler les caractéristiques principales du régime d'accumulation coréen dans une première période (1960-1987) qui est celle de la sortie du sous-développement. L'accent sera mis, en particulier, sur les interrelations entre les changements structurels qui rythment l'industrialisation accélérée de la Corée et le rapport salarial. Nous mettrons en évidence à quel point la flexibilité quantitative du travail a été déterminante pour le succès de l'industrialisation mais aussi, dans certaines branches industrielles, pour le développement de la flexibilité fonctionnelle, à un coût relativement faible.

La $2^{\mathrm{e}}$ section s'attachera ensuite à montrer comment ce régime d'accumulation rencontre des limites sociales, l'année 1987 étant de ce point de vue une année charnière. La démocratisation de la société et la contestation sociale qui l'accompagne imposent des limites à la flexibilité quantitative. La flexibilité fonctionnelle ne peut alors se développer qu'à un coût plus élevé, ce qui pèse sur la compétitivité des entreprises. Ce problème est d'autant plus crucial que l'ouverture croissante de l'économie à la concurrence étrangère impose aux entreprises coréennes une contrainte de rentabilité à laquelle elles sont peu habituées. La crise financière de l'été 1997

1. Cf. R. Boyer (dir.) (1986). La flexibilité du travail en Europe, Paris, Éd. La Découverte, p. 236 et s.

2. Cf. B. Brunhes (1989), La flexihilité du marché du traicail. Paris, OCDF. 
renforce cette contrainte et précipite l'adoption de révisions de la législation du travail accroissant la flexibilité quantitative qui jusqu'alors avaient été refusées par les syndicats coréens. L'impact sur la flexibilité fonctionnelle est un des enjeux de la période à venir.

UN REGIME D'ACCUMULATION ORRGINAL.

\section{Une croissance dirigée par l'État}

L'économie coréenne attire l'attention par l'ampleur de sa croissance et sa rapiditél. Au cours des trente dernières années, le PIB réel a été multiplié par 12 et le revenu par habitant par 7 . La croissance s'est accélérée au début des années 1960 après l'adoption d'une stratégie d'industrialisation fondée sur la promotion des exportations qui, à la fin des années 1980, représentent $35 \%$ du PIB. Aujourd'hui, le PIB de la Corée est de l'ordre de $40 \%$ de celui de la moyenne de l'OCDE et, si le rythme actuel de croissance était maintenu, le PIB par tête atteindrait $20000 \$$ par tête en 2001, soit un niveau équivalent à celui de l'Espagne ou du Canada $^{2}$. Ce rythme de croissance accéléré a eu pour conséquence une baisse régulière du taux de chômage et une progression rapide du salariat qui devient majoritaire après 1983, l'agriculture n'occupant plus que $15 \%$ de la population active en 1994. A la fin des années 1980, le plein-emploi était atteint.

L'origine de la croissance coréenne, et de celle des autres NPI de la première génération, est objet de controverse. Pour ce qui concerne la seule Corée, G. Ranis et J. Frei, et B. Balassa ${ }^{3}$ estiment que l'entrée dans la période de croissance accélérée à partir de 1965 s'explique avant tout par le passage d'une industrialisation tirée par la substitution d'importations à une industrialisation tirée par les exportations, accompagné par des réformes structurelles visant à promouvoir les mécanismes de marché, comme la réduction du protectionnisme, l'adoption de taux de change réalistes et de taux d'intérêt réels positifs ${ }^{4}$. Cette explication

1. Entre 1963 et 1990 , le taux de croissance annuel moyen du PlB en Corée atteint $8.7 \%$ contre $3.7 \%$ pour la moyenne de l'OCDF, of. Études économiques de l'OCDE. Paris. OCDE, 1996 a.

2. Cf. B. Brunhes (1989), La flexibilite du marché du travail. Paris, OCDE, p. 14.

3. Cf. G. Ranis. J. Frei (1975). A model of growth and employment in the open dualistic economy: the cases of Korea and Taiwan, in F. Stewart (ed.). Emplovment, Income Distribution and Development, London, Frank Cass: B. Balassa (1982), Development strategies and Economic Performance, in B. Balassa (ed.) ct al. Development Strategies in Semi-Industrial Economies, Baltimore. The John Hopkins University Press.

4. McKinnon R. (1991). The Order of Economic Liheralization. Baltimore and London. The John Hopkins University Press. 
est contestée sur le plan factuel par $\mathrm{R}$. Luedde-Neurath' qui montre que les restrictions aux mécanismes de marché sont restées extrêmement fortes au cours des années 1960-1980 et que, parallèlement à la promotion des exportations qui débute dans les années 1960, la substitution d'importations à l'abri des barrières douanières a continué à être soutenue par l'État, si bien qu'il est difficile de voir dans l'accélération de la croissance la manifestation de l'efficience des marchés. Une deuxième explication portée par $\mathrm{J}$. Bhagwatti et la Banque mondiale ${ }^{2}$ reconnaît que l'État coréen a continué d'intervenir fortement dans l'économie mais, volontairement ou non, il l'a fait en respectant les marchés. Dans le domaine du commerce international, des mesures contradictoires auraient abouti à une structure d'incitation neutre, créant un régime de "libre-échange virtuel». Par ailleurs, et d'une manière plus générale, l'État coréen aurait été plus "prescriptif » que "proscriptif», c'est-à-dire aurait plus incité qu'interdit, laissant ainsi plus de liberté à l'initiative privée. Cette deuxième explication a aussi été réfutée, tant sur le plan théorique qu'empirique ${ }^{3}$, si bien que l'on doit accepter l'idée que la Corée est un exemple d'intervention efficace de l'État dans le processus de développement ${ }^{4}$. Parmi les nombreux facteurs historiques, économiques et politiques qu'il faudrait mentionner pour étayer cette explication alternative, on en retiendra un, qui distingue la Corée des autres expériences de développement: la formation d'un État très fortement autonome, non seulement du salariat ${ }^{5}$, mais aussi des entrepreneurs industriels ${ }^{6}$. Il en est résulté une capacité à formuler une politique de développement articulée et cohérente, s'imposant aux intérêts privés, et/ou créant des intérêts privés compatibles avec l'application de cette politique. L'État coréen va ainsi favoriser l'émergence de conglomérats industriels privés très puissants (les Chaebols), qui lui seront longtemps

1. Cf. R. Luedde-Neurath (1986), Import Controls and Export-Oriented Development; a Reassessment of the South Korean Case, Boulder and London, Westview Press.

2. Cf. J. Bhagwatti (1987), Outward Orientation: Trade Issues, in V. Corbo, M. Khan, M. Goldstein (eds), Growth Oriented Siructural Adjustment, Washington DC, IMF and World Bank; Banque mondiale (1993), The East Asian Miracle, Economic Growth and Public Policy, Washington.

3. On trouvera un exposé des arguments théoriques et empiriques conduisant à rejeter la thèse de l'intervention de l'État préservant le marché dans H. J. Chang (1993), The Political Economy of Industrial Policy in Korea, Cambridge Journal of Economics, vol. 17, p. 131-157.

4. Cf. L. Jones, I. Sakong (1980), Government, Business and Entrepreneurship in Economic Development: The Korean Case, Cambridge, Harvard University Press; A. Amsden (1989), Asia's next giant. New York, Oxford University Press; H. J. Chang (1993), op. cit.

5. Lune des conséquences de la guerre de Corée (1950-1953) sera laffaiblissement durant la majeure partie de la période d'industrialisation rapide (1960-1987) du syndicalisme militant et combatif, qui sera interdit au profit d'un syndicalisme officiel inféodè à l'État.

6. H. J. Chang (1993, op. cit.) rappelle un fait significatif: au début des années 1960 , le régime militaire du général Park Chung Hee avait emprisonné des chefs d'entreprise sous l'accusation d' "enrichissement illicite ", puis il les relâcha contre la promesse de «servir la Nation grâce à l'entreprise ". c'est-à-dire l'investissement dans les industries retenues comme étant prioritaires par le plan. 
subordonnés par l'intermédiaire du système bancaire, nationalisé en 1961, qui organise le rationnement des crédits en leur faveur. Les Chaebols seront les fers de lance de la mise en œuvre de la planification économique. Leur diversification croissante dans des secteurs industriels nouveaux a été voulue par l'État qui y voyait le moyen de créer ou de renforcer les industries jugées prioritaires par le plan'. Il en résultera une structure industrielle très concentrée, organisée autour d'une dizaine de grands groupes horizontalement intégrés ${ }^{2}$, au taux d'endettement très élevé, mais capables de réaliser des économies d'échelle grâce à leur dimension et à la priorité accordée à l'exportation massive de leur production.

Cette intervention efficace de l'État dans le processus d'accumulation se concrétisera par un rythme d'industrialisation beaucoup plus rapide que la moyenne des pays en développement. Mais surtout il permettra d'opérer avec succès une mutation de l'industrie qui, initialement spécialisée dans les biens de consommation légers, devient de plus en plus spécialisée dans les biens intensifs en capital et en technologie. La réussite est particulièrement marquée dans le domaine des industries lourde et chimique, dont le développement est décidé et organisé à partir de 1973 et qui, dès 1979, représentent $55 \%$ de la production industrielle. Phénomène remarquable, leur part dans les exportations dépasse les $50 \%$ au début des années 1980 et progresse ensuite régulièrement pour atteindre $60 \%$ au début des années 1990 .

Cette évolution de la structure industrielle entraîne une évolution de la demande de travail des entreprises. Le procès de travail dans les industries intensives en capital requiert une force de travail aux caractéristiques différentes. Un faible coût salarial et une productivité élevée du travail ne suffisent plus. Une coopération minimale des salariés est indispensable à l'efficacité productive. La réunion de ces conditions suppose un rapport salarial adéquat. Dans ce domaine aussi, l'intervention de l'État s'avérera décisive.

1. M. Lautier écrit à ce propos : «L’interventionnisme et le soutien sélectif aux grands groupes se sont renforcés lors des troisième et quatrième plans quinquennaux (1972-1981) qui ont pour objectif la promotion des industries lourdes et chimiques. L'État désigne alors précisément les secteurs à promouvoir et les entreprises qui en ont la charge. Si l'investissement dans ces activités plus intensives en capital est réalisé par des groupes privés, le financement est pour l'essentiel public. Une entreprise qui s'engageait dans un secteur prioritaire finançait seulement $20 \%$ du projet sur ses fonds propres et obtenait le complément de l'État. directement ou par l'intermédiaire des banques " (M. Lautier (1997). Dynamiques des structures industriclles et développement. thèse pour le doctorat de Sciences économiques. Université Pierre-Mendès-France - Grenoble II, miméo. p. 451.

2. Les dix premiers chaebols représentaient $22,5 \%$ de la valeur ajoutée manufacturière en 1989 selon M. Lautier (1997, op. cit., p. 440), soit beaucoup plus que la moyenne des pays développés et des pays en développement. Elle s'est renforcée depuis, selon la Korea Fair Trade Commission.

3. Cf. OCDE, 1996 a. op. cit., p. 25-28. 


\section{La flexibilité au cœur du rapport salarial coréen}

L'intervention de l'État dans le domaine de la détermination de l'emploi, des salaires, de la productivité du travail, est souvent occultée ou dénoncée par les approches théoriques qui privilégient une analyse en termes de marché où se confrontent une offre et une demande de travail. Un marché du travail fonctionnant librement conduit au pleinemploi grâce à la flexibilité (quantitative) des salaires réels et du travail. Dans le cas des pays en développement, le problème se pose autrement. L'existence initiale d'un excédent de main-d'œuvre (c'est-à-dire une offre parfaitement élastique) conduit à envisager, à la suite de la théorie dualiste du marché du travail', une première phase de stabilité du salaire industriel. Dans une deuxième phase, où l'accélération de la croissance doit conduire à l'épuisement de l'excédent et à la transformation du travail en facteur rare, le salaire réel augmentera, car il devient un indicateur de rareté, comme dans les pays développés. Si le marché du travail opère librement, un taux de salaire d'équilibre se réalisera.

\section{Analyse et répression du marché du travail}

Dans le cas de la Corée, où la croissance s'est accélérée grâce aux exportations des industries intensives en travail, l'épuisement progressif de l'excédent de main-d'œuvre justifie, pour certains, une intervention de l'État visant à empêcher ou à limiter les hausses de salaire à un niveau inférieur au taux d'équilibre du marché. Ceci, afin de préserver l'avantage des bas salaires qui détermine la compétitivité-coût des exportations de biens souvent fortement substituables. Autrement dit, la répression du travail serait un mal nécessaire à la sortie du sous-développement.

G. Fields conteste ce point de vue ${ }^{2}$. Toute mesure excessive de restriction à la hausse des salaires (répression salariale) et de restriction à l'organisation syndicale des salariés (répression du travail) ${ }^{3}$ peut conduire à des problèmes équivalents à ceux d'un taux de salaire supérieur au taux d'équilibre. Lorsque le salaire est réprimé, au point où il devient inférieur à

1. Cf. A. W. Lewis (1954), Development with Unlimited supplies of Labour. Manchester School, vol. 22 (May), p. 139-191

2. G. Fields (1994), Changing Labor Market Conditions and Economic Development in Hong-Kong, the Republic of Korea, Singapore, and Taiwan, China, The World Bank Economic Review, vol. 8, n" 3 , p. $395-414$.

3. G. Fieids (1994, op. cit., p. 403) emploie l'expression de "répression du marché du travail ", qui regroupe à la fois la répression des salaires et la répression du travail (qui vise les organisations syndicales), pour désigner les discriminations exercées par l'État à l'encontre de l'offre de travail, ce qui n'est pas sans rappeler la "répression financière 》 définie par $\mathrm{R}$. McKinnon (1991, op. cit.) à propos des restrictions imposées par l'État à l’offre de capital sur les marchés financiers dans les PVD. 
son niveau d'équilibre, on assiste à une baisse de l'offre de travail. Il en résulte une croissance inférieure à celle qui pourrait être atteinte si le marché du travail pouvait s'équilibrer. De la même manière, lorsque le pouvoir de négociation des salariés est trop fortement réduit, la part des salaires dans le revenu national sera trop faible, conduisant à une demande insuffisante sur le marché des biens et services.

Cette analyse est mobilisée pour rendre compte de l'expérience des NPI d'Asie du Sud-Est. Singapour aurait traversé une période de répression des salaires (1972-1979) ayant réduit l'emploi et la croissance. Mais aucun des quatre "dragons " n'aurait connu sur le long terme de répression du travail significative, certains allant jusqu'à conclure que, même en Corée, les conditions du marché du travail auraient été semblables à celles d'un marché concurrentiel, et les salaires auraient été proches du niveau d'équilibre'. Ce jugement concernant la Corée s'appuie sur le constat suivant: la répression du travail a bien eu lieu², mais n'a sans doute pas réellement atteint ses objectifs puisque: 1/les salaires réels ont augmenté sur le long terme (1965-1985) plus rapidement que la productivité ; 2 / le taux de chômage n'a cessé de baisser, le plein-emploi étant pratiquement réalisé dans la deuxième moitié des années 1980 ; 3 / la part des revenus du travail dans le revenu national est passée de $31,8 \%$ en 1965 à $51,6 \%$ en 1980 et $54,3 \%$ en $1987^{3}$.

Cette analyse est intéressante en ce qu'elle montre à sa manière que l'amélioration du niveau de vie est compatible avec la sortie du sousdéveloppement. Mais l'idée selon laquelle ce résultat favorable serait la manifestation de l'existence d'un marché du travail proche d'un marché concurrentiel est critiquable et trompeuse. Critiquable car, sur le plan théorique, outre les problèmes éthiques et moraux en cause, elle revient à supposer que l'intervention de l'État, en l'occurrence un taux de répression du travail optimal, permettrait d'atteindre un taux de salaire d'équilibre équivalent à celui qui serait réalisé si le marché du travail fonctionnait de façon totalement concurrentielle, ce qui reste à démontrer. Trompeuse, car les faits invoqués à l'appui de la démonstration, bien qu'indéniables, ne suffisent pas à rendre compte d'une réalité bien plus complexe :

- Sur l'ensemble de la période d'industrialisation rapide (19631988), il est vrai que les salaires réels ont crû à un rythme très élevé $(8,1 \%)$, supérieur aux gains de productivité du travail dans l'industrie

1. G. Fields, H. Wan (1989), Wage Setting Institutions and Economic Growth, World Development, vol. 17, n" 9. p. 1471-1484, et G. Fields (1994), op. cit.

2. Concernant l'attitude de l'État coréen à l'égard des syndicats indépendants, G. Fields (1994, op. cit.) emploie lexpression explicite de smoking gun approach.

3. En 1996. cette part est de $64,7 \%$, soit un niveau équivalent à celui de la moyenne des pays de IOCDE $(65,2 \%)$; cf. OCDE, 1996 a, op. cit. 
$(6,8 \%)^{1}$. Cependant, de 1965 à 1973, période où prédominent les industries intensives en travail, la croissance des gains de productivité du travail est supérieure $(13 \%)$ à celle des salaires réels $(9,8 \%)$. Ce n'est qu'avec le développement de l'industrie lourde, à partir de 1973, que les salaires réels augmentent tendanciellement plus vite que les gains de productivité. Selon A. Amsden², cette hausse des salaires ne peut s'expliquer uniquement par l'analyse de l'offre et de la demande de travail. Elle dépend aussi du contexte institutionnel et social dans lequel opèrent les forces du marché. L'État, à certaines périodes, a exercé des pressions sur les entreprises afin qu'elles augmentent les salaires. C'était pour lui une source de légitimation et de paix sociale, compensant les périodes de forte répression du travail ${ }^{3}$. La hausse des salaires s'explique aussi, selon nous, par la combativité des salarié(e)s qui, bien que réprimé(e)s, n'ont jamais cessé, au cours des années 1970, de revendiquer des hausses de salaires ${ }^{4}$.

- Par ailleurs, cette hausse des salaires n'a pas bénéficié à tout le monde dans les mêmes proportions. Les femmes ont subi une discrimination sur le marché du travail plus élevée que partout ailleurs. En 1980 , le salaire moyen des femmes représentait $44,4 \%$ de celui des hommes, niveau le plus faible enregistré à l'échelle internationale, à l'exception du Japon où le degré de discrimination est comparable, mais bien loin derrière la France, où le salaire moyen des femmes atteignait $75 \%$ de celui des hommes. L'emploi féminin étant principalement concentré dans les industries intensives en travail (notamment celles localisées dans les zones franches), cette discrimination a contribué à maintenir la compétitivité-coût des entreprises travaillant pour l'exportation. Les hausses de salaires ont essentiellement bénéficié aux salariés des industries intensives en capital, mieux à même de défendre leurs intérêts, et dont le procès de travail nécessite des formes d'incitation plus motivantes.

1. Cf. G. Fields (1994, op. cit., p. 405); M. Fouquin et al. (1991), Pacifique : le recentrage asiatique, CEPII, Paris, Economica, p. 84.

2. 1989, op. cil.. p. 198-200.

3. Au total, la hausse des salaires ouvriers sera plus forte que celle des salariés de qualification plus élevée. Il s'agit là d'un trait spécifique à la Corée qui contraste fortement avec l'expérience d'autres pays en développement rapide, notamment en Amérique latine. Au Brésil, par exemple, où l'État est lui aussi très présent dans la formation des salaires, les gains de productivité élevés obtenus durant la période du " miracle économique " (1967-1973) ont surtout bénéficiè aux cadres dirigeants, qui ont obtenu parfois des hausses de revenus réels supérieures aux gains de productivité. tandis que la grande majorité des salariés, notamment les moins qualifiés, obtenait des hausses de salaires réels inférieures, cf. J. Sabóia (1987), Teoria da Regulação e “Rapport Salarial » no Brasil, Texto para discussão, n"143, Instituto de Economia Industrial, Universidade Federal do Rio de Janeiro, p. 22-24.

4. Cf. W. Bello, S. Rosenfelf (1990), Dragons in Distress. Asia's Miracle Economies in Crisis, San Francisco, Food first. 


\section{La répression du travail a permis l'essor de la flexibilité quantitative}

Il faut donc aller au-delà du simple constat de la hausse sur le long terme des salaires réels pour découvrir la fonctionnalité réelle de la répression du travail. En l'occurrence, la répression du travail exercée par l'État coréen a permis, au sein des entreprises, une gestion de la force de travail que l'on qualifiera, à la suite de J. I. You', de "paternalisme autoritaire». Paternaliste car devant la faiblesse, à cette époque, de la protection sociale ${ }^{2}$, les entreprises accordent à leurs salariés, dans des proportions variables et de manière discrétionnaire, des avantages non salariaux dans le domaine du logement, de la santé, de l'éducation, dans l'objectif d'instaurer une dépendance envers l'entreprise, de la faire apparaître comme bienveillante et de créer un climat de coopération et de loyauté. Autoritaire, car les conditions d'emploi extrêmement dures qui l'accompagnent conduisent les entreprises à recourir à l'arbitraire pour imposer une stricte discipline ${ }^{3}$. Répression du travail et paternalisme autoritaire vont créer un rapport salarial qui fait de la flexibilité quantitative une de ses caractéristiques principales. Cette flexibilité quantitative, qui est donc bien loin d'être le produit spontané du marché, s'appuie sur les éléments suivants :

La flexibilité des salaires réels. La hausse des salaires réels sur le long terme n'a pas entraîné l'émergence d'une rigidité à la baisse. Plusieurs éléments concourent à cette flexibilité. Tout d'abord l'absence de salaire minimum jusqu'en 1988 et de mécanismes d'indexation sur la productivité. Ensuite, le faible impact des syndicats dans la détermination des salaires durant la majeure partie de la phase d'industrialisation rapide, les syndicats étant parfois légalement interdits de participation aux négociations salariales comme au début des années 1980 , période de répression intense du travail. Enfin, en Corée comme au Japon, la part variable du salaire est élevée ( $30 \%$ en Corée), ce qui permet d'ajuster les salaires à la conjoncture comme le montrent les estimations de

1. Cf. J. I. You (1995), Changing Capital-Labour Relations in South Korea, in J. Schor, J. I. You (eds), Capital, the State and Labour. A Global Perspective, Aldershot, GB, Brookfield, Etats-Unis, Edward Elgar \& The United Nations University Press. p. 121.

2. La part des dépenses sociales dans les dépenses totales de l'État est de 7,5\% sur la période 19721985, contre 27,2\% dans les PVD à revenu élevé et 46,7\% dans les pays développés d'après la Banque mondiale (1993, op. cir.).

3. L'Etat collabore avec les entreprises dans cette démarche, en tentant de développer le mouvement Saemaul, avec des mots d'ordre tels que : "Travailler dur sans être conscient de l'heure qui passe " ou bien : "Les travailleurs doivent se comporter vis-à-vis de leur employeur comme les enfants vis-à-vis de leurs parents. "A ce sujet voir W. Bello, S. Rosenfeld (1990. op. cit.). A un autre niveau, on rappellera que les services de renseignements sont officiellement en charge des questions sociales à cette époque. 
M. Lanzarotti ${ }^{1}$. Ce salaire variable comprend les primes (appelées "bonus »), fonction des résultats de l'entreprise, et le paiement des heures supplémentaires. Ces dernières, dans la pratique, sont souvent payées au même taux que les heures régulières et parfois sous la forme d'un forfait, indépendamment du nombre d'heures effectuées ${ }^{2}$. Tous ces éléments ont permis, durant les périodes de stagflation, de restaurer la compétitivité-coût grâce à des baisses de salaires réels.

La flexibilité horaire du travail. Les hausses de salaires trouvent leur contrepartie dans une durée et une intensité du travail inégalées. La durée hebdomadaire du travail dans l'industrie manufacturière était de 54,4 heures en 1960, comparable à la situation prévalant à Taiwan, mais bien plus qu'au Japon (47,8 heures), en Thaillande (47,1 heures) et aux Philippines (44,2 heures) pour la même année. En 1985, elle était encore de 53,8 heures, soit un niveau nettement supérieur aux autres pays d'Asie. ${ }^{3}$. Elle était plus élevée pour les femmes que pour les hommes et pour les manuels que pour les autres professions. Cette durée hebdomadaire élevée s'explique en partie par des heures supplémentaires particulièrement longues $(7,5$ heures supplémentaires en moyenne s'ajoutant à une durée régulière hebdomadaire de 44,4 heures sur la période 1980$1988)^{4}$. Cette situation est facilitée par la législation du travail qui autorise de nombreux dépassement des horaires pour adapter le temps de travail au volume de la production.

La flexibilité de l'emploi. La législation coréenne du travail durant cette période prévoit que les employeurs ne peuvent licencier sans «raisons justifiables", sans que pour autant celles-ci soient précisément définies. Dans la pratique, les grandes entreprises, et plus encore les PME où les syndicats sont inexistants, ont pu licencier sans contraintes réelles et ajuster le volume de l'emploi au volume de la production ${ }^{5}$. Selon J. I. You ${ }^{6}$, qui montre que les grandes entreprises ont réduit plus fortement leurs effectifs que les PME durant la récession de 1980-1981, ce recours aux licenciements distingue nettement la Corée du Japon où les grandes entreprises avaient recherché l'implication de leurs salariés en leur accordant un certain nombre d'avantages sociaux comme parfois la sécurité de l'emploi. En Corée, les conditions d'emploi ont souvent

1. Cr. M. Lanzarotti (1992), La Corée du Sud: une sortie du sous-développement, Paris, IEDES/PUF, p. $220-222$

2. Cf. Y. K. Park (1979), Labor and the Business Environment in Korea. Asian Economies, December, n०31, p. 31.

3. Sources : Korean Labor Institute (1993), Labor in Korea, Séoul ; BIT, Rapport annuel des statistiques du travail, Genève : Fouquin et al, op. cit.

4. Source : Korean Labor Institute (1993), Labor in Korea, Séoul, p. 66

5. Cf. Y. K. Park, 1979, op. cit., p. 28-29.

6. Op. cit., p. 130. 
conduit à une usure prématurée de la force de travail qui n'était généralement pas compensée par des contreparties sociales. En conséquence, les salariés coréens n'ont pas hésité à quitter leur entreprise dès lors qu'un emploi meilleur se présentait, ce qui est d'autant plus fréquent en période de croissance accélérée. Il en est résulté une forte mobilité de l'emploi se matérialisant, durant les années 1970, par un taux mensuel de rotation du travail (mesuré par le taux de démission) plus élevé en Corée $(5 \%)$ qu'aux États-Unis $(4 \%)$ et au Japon $(1,5 \%)$. Ce taux mensuel de rotation était en 1985 nettement plus élevé dans les industries intensives en travail $(6,5 \%)$ que dans les industries intensives en capital $(3,5 \%)$.

L'ensemble des caractéristiques de la flexibilité quantitative qui viennent d'être évoquées indique une forte segmentation du marché du travail opposant les industries intensives en travail à celles intensives en capital, les petites et les grandes entreprises. C'est dans ce contexte que l'on assiste à un développement limité de la flexibilité fonctionnelle. Dans les industries intensives en capital, notamment les industries en continu, A. Amsden' soutient que les directions d'entreprises ne connaissaient initialement pas grand-chose aux technologies qu'elles importaient, ce qui les rendaient très dépendantes de l'implication des ouvriers dans la mise au point et l'amélioration continuelle des équipements importés. Cette implication a été obtenue grâce à des contreparties visant à motiver les salariés sous la forme de rémunérations plus élevées que dans les autres secteurs, et souvent par une sécurité de l'emploi de fait. Des compétences spécifiques, un savoir tacite ont pu ainsi s'accumuler et se partager au service d'un apprentissage organisationnel rapide. Ce qu'il est important de souligner, c'est que les salaires versés sont élevés relativement à des salaires moyens qui, eux, sont d'un niveau très faible, et que la sécurité de l'emploi est d'autant plus appréciée qu'elle n'existe pas partout. C'est en ce sens que la flexibilité fonctionnelle est étroitement dépendante de la flexibilité quantitative. Les contreparties accordées ne sont pas onéreuses en termes absolus; du reste, elles ne concernent pas toutes les caractéristiques de l'emploi. La semaine normale des sidérurgistes de l'entreprise POSCO, citée en exemple par A. Amsden ${ }^{2}$, est de 56 heures ( 8 fois 7 heures), dont 45 heures régulières et 11 heures supplémentaires payées au même tarif. Une fois maîtrisées les technologies importées, POSCO réunissait ainsi toutes les conditions pour être compétitive. 
Au total, derrière l'image globale d'une hausse de salaire réel supérieure en tendance aux gains de productivité, se dissimule un marché du travail très fortement segmenté, qui permet de comprendre comment la compétitivité-coût des exportations a pu être relativement préservée.

Un régime d'accumulation " reposant sur l'exportation et la consommation non durable"

Cette caractérisation proposée par $\mathbf{M}$. Lanzarotti ${ }^{1}$ permet de souligner les spécificités de l'industrialisation coréenne. La répression du travail et le paternalisme autoritaire ont contribué à la création d'une industrie intensive en travail, compétitive, capable de dégager des gains de productivité élevés par des moyens archaïques : durée et intensité du travail élevées. Ces gains de productivité ont permis d'accorder des hausses de salaires, qui ont engendré pour l'essentiel une demande de biens de consommation non durables importante, et une épargne élevée, qui prend le caractère d'une épargne contrainte. En effet, la demande de biens de consommation durables a longtemps été limitée par le gouvernement de façon à contraindre les ménages à épargner ${ }^{2}$. De cette manière, une partie non négligeable du surplus créé par les industries d'exportation intensives en travail a été canalisée vers les banques (publiques) qui l'ont utilisée pour accorder des crédits à taux faibles aux entreprises investissant dans l'industrie lourde et chimique. Grâce à ce mécanisme, les bas salaires ont été transformés en capital à bon marché. La question qui se pose cependant est de savoir dans quelle mesure ce type d'industrialisation a été propice à un rattrapage technologique. Le développement d'une industrie lourde ne suffit pas à répondre à la question dans la mesure où il ne suffit pas de constater l'augmentation de l'intensité capitalistique mais d'évaluer avec quelle efficacité travail et capital sont utilisés. A ce sujet deux thèses s'opposent. A. Amsden ${ }^{3}$ soutient que la politique industrielle poursuivie en Corée a favorisé un processus de rattrapage par importation des technologies étrangères, et que le maintien du protectionnisme a donné au processus de learning by doing le temps nécessaire à une imitation réussie, puis à la mise en place de processus d'apprentissage durable. A l'opposé P. Krugman ${ }^{4}$, s'ap-

1. Op. cit., p. 191 .

2. H. J. Chang (1993,op. cit., p. 138) en donne des exemples: il était demandé aux banques publiques de ne pas accorder de crédit à la consommation. La taxation indirecte visait à décourager la consommation. Les vacances à l'étranger étaient interdites jusqu’à la fin des années 1980 , et les importations de biens étrangers " de luxe " interdites ou soumises à une taxation prohibitive.

3. Op. cit. p. 20-21.

4. P. Krugman (1994), The Myth of Asia's Miracle. Foreign Affairs, November/December. 
puyant sur des estimations du modèle néo-classique de la croissance réalisées par A. Young', estime que le fameux "miracle asiatique" s'explique simplement par deux éléments. Une mobilisation particulièrement réussie de la population active et du capital disponible, comme l'économie soviétique avait aussi réussi à le faire dans les années 1950 ; et une augmentation rapide d'une offre de travail de plus en plus qualifié et surtout du taux d'investissement. Le taux d'investissement et la qualification ne pouvant augmenter éternellement, P. Krugman en conclut que le rythme de croissance devrait bientôt ralentir. Sans entrer dans le détail de la discussion théorique, et notamment le fait que la productivité globale ${ }^{2}$ soit considérée comme un résidu inexpliqué, force est de constater que, dans le cas de la Corée, les résultats obtenus par A. Young montrent que la productivité totale des facteurs n'est pas si négligeable. Pour ce qui concerne l'ensemble de l'économie, la productivité totale des facteurs est de 1,7\% sur l'ensemble de la période 19661990 , avec une accélération notable en fin de période $(2,6 \%$ dans la deuxième moitié des années 1980). Pour l'industrie manufacturière, la productivité totale fluctue fortement d'une décennie à l'autre en fonction des vagues d'investissements qui correspondent au passage de l'industrie légère à l'industrie lourde. Mais, sur l'ensemble de la période 1966-1990, le taux de croissance annuel moyen de la productivité totale atteint le niveau élevé de $3 \%$. Autrement dit il y aurait eu, dans l'industrie coréenne, de réels gains d'efficacité révélateurs d'un processus de rattrapage et pas seulement une capacité exceptionnelle à mobiliser des ressources. Ces chiffres confirment, selon nous, l'importance des processus d'apprentissage qui ont permis progressivement à la Corée d'imiter avec succès les techniques de production de masse mises au point dans les pays développés. La question qui se pose est de savoir si elle saura renouveler cette performance dans le cadre d'une société démocratisée et au moment où les modèles socioproductifs sont en train d'évoluer.

DÉMOCRATISATION DE LA SOCIÉTÉ ET FLEXIBILITÉ DU TRAVAIL

1987 est une année de rupture pour la Corée en ce sens qu'elle inaugure un processus de démocratisation de la société qui débouchera sur la tenue d'élections présidentielles au suffrage direct en 1992. Elle est marquée par un mouvement gréviste sans précédent qui ébranle l'ancien

1. A. Young (1995), The Tyranny of Numbers : Confronting the Statistical Realities of the East Asian Growth Experience, The Quaterly Journal of Economics. August. p. 642-680.

2. La productivité globale incorpore à la fois le progrès technique et ce que l'on pourrait appeler le progrès organisationnel. c'est-à-dire une combinaison plus efficace du travail et du capital. 
système de répression du travail et conduit à l'adoption d'une nouvelle législation du travail plus permissive. Plus généralement, la démocratisation annonce un mouvement lent et graduel de retrait de l'État dans la sphère économique et sociale. Les conditions politiques et sociales qui avaient conduit à une forte dualisation du marché du travail et à une flexibilité quantitative élevée s'en trouvent remises en cause, ce qui va contraindre les entreprises à revoir leurs stratégies. Le rééquilibrage des forces sociales en faveur des salariés va conduire à une atténuation, pour une partie d'entre eux, des aspects les plus contestés de la flexibilité quantitative, et à une progression très importante du salaire réel. La flexibilité fonctionnelle, qui repose sur des contreparties accordées aux salariés, en subira aussi les contrecoups. La compétitivité-coût des entreprises coréennes va ainsi se dégrader pour des raisons internes au moment où la Corée s'engage dans un processus graduel d'ouverture économique et financière qui va accroître les tensions qui pèsent sur le régime d'accumulation. Tels sont les points que nous allons développer.

\section{La remise en cause du rapport salarial}

Les réformes de 1987 ont deux impacts immédiats : l'accroissement brutal de l'activité gréviste' et le triplement du nombre de syndicats. Dans les années qui suivent, le nombre de conflits du travail diminue fortement $t^{2}$ et les effectifs syndicaux régressent, ce qui s'explique en partie par la baisse de la part relative de l'emploi manufacturier où les syndicats sont les mieux implantés. Le taux de syndicalisation, qui avait dépassé les $20 \%$ en 1988-1989, tombe à $14,5 \%$ en 1994 , soit un niveau inférieur à bon nombre de pays de l'OCDE, mais supérieur à celui de la France (12\%) et proche de ceux des États-Unis et de l'Espagne $(16 \%)$.

Cette baisse de la conflictualité ne signifie pas pour autant un retour en arrière. Une nouvelle législation du travail améliore le respect de certaines des «normes fondamentales du travail $»^{3}$ qui ne l'étaient pas auparavant, comme la liberté syndicale et le droit de négociation collec-

1. Il y a eu autant de grèves entre juillet et août 1987 que durant les vingt-cinq années précédentes.

2. Le nombre de conflits sociaux officiellement enregistrés est de 276 en 1986 . Il culmine à 3749 en 1987. En 1995, il est de 88. Le nombre de jours de travail perdus est de 7200 en 1986, 6947 en 1987 , et 393 en 1995 (source : OCDE, 1996 a, op. cit., p. 114-116).

3. Selon le BIT, sont considérées comme "normes fondamentales du travail » les libertés suivantes: la liberté syndicale et le droit à la négociation collective, l'interdiction du travail forcé et l'abolition du travail des enfants. Ces normes ont été consacrées par le Sommet mondial pour le développement social, Copenhague, 1995. L'OCDE (1996 b, p. 28) y ajoute l'absence de discrimination dans l'emploi, c'est-à-dire le droit de tous les travailleurs au même respect et au même traitement. Le respect de ces normes est devenu un enjeu des discussions commerciales internationales (OCDE (1996 b). Normes du travail, commerce et emploi, Paris, OCDE). 
tive, même si pour autant toute forme de répression du travail n'a pas disparu, ce qui a entraîné les critiques de l'ort, lors de l'adhésion de la Corée en 1991 (encadré).

Malgré ces restrictions maintenues, les salariés ont pu obtenir les améliorations suivantes :

- De fortes augmentations des salaires réels de 14,7\% entre 19901995, supérieures aux gains de productivité, 8,5\%'. Ces augmentations ont surtout profité aux salariés des grandes entreprises (plus de 500 salariés) où le taux de syndicalisation est le plus élevé, plutôt qu'à ceux des PME (moins de 30 salariés). La différence entre les salaires est passée de $11 \%$ en 1986 à $38 \%$ en 1994. Les conflits se sont ensuite déplacés sur d'autres sujets tels que la durée du travail, les conditions de travail, la participation des salariés à certaines décisions, l'introduction dans la négociation collective de questions nouvelles. Dans un certain nombre de domaines qui concernent la flexibilité quantitative, les évolutions ont été importantes.

\section{Le respect des normes du travail en Corée}

- Le droit de créer des syndicats libres

«La loi n'autorise qu'un seul syndicat par entreprise. Cependant il n'est pas fait état de plaintes arguant que cette exigence limite la liberté syndicale. Il existe de sérieuses limitations à la création de fédérations et de confédérations nationales. Une seule confédération nationale a été reconnue jusqu'à présent. »

- Le droit de grève

«La loi interdit l'intervention d'une tierce partie dans les conflits du travail. Dans la pratique, les syndicats indépendants (non reconnus) se voient souvent refuser le droit d'intervenir dans un conflit. En cas de non-respect de ces dispositions, ce qui arrive fréquemment, les grévistes sont licenciés et les leaders syndicaux arrêtés. Le droit de grève est soumis à une notification préalable et au respect d'un délai "de réflexion" de dix jours. La grève est interdite dans les industries de la défense."

- La protection des travailleurs syndiqués et du droit de négociation collective

"Les travailleurs des "fédérations et confédérations non enregistrées n'ont pas à intervenir dans les activités syndicales au niveau de l'entreprise, y compris dans les négociations collectives (interdiction de l'intervention d'une tierce partie). Certains adhérents de syndicats non enregistrés ont fait l'objet de poursuites judiciaires liées à l'intervention d'une tierce partie. "

Source : Normes du travail, commerce et emploi, OCDE, 1996 b, p. 62-73.

1. Calculs personnels d’après les statistiques de la comptabilité nationale (Bank of Korea) et de l'Institut coréen du travail (KLI). 
- Dans le secteur non agricole, la durée hebdomadaire effective du travail, qui avait culminé à 52,4 heures en 1986 , est passée à 47,4 heures en 1994, les heures supplémentaires étant ramenées de 7,9 à 5,8 heures'. La réduction du temps de travail a été plus forte pour les femmes, la durée hebdomadaire des hommes et des femmes est maintenant comparable. Dans l'industrie manufacturière, la durée hebdomadaire en 1995 (49,2 heures) reste malgré tout la plus élevée des pays de l'OCDE et supérieure à celle de Taiwan.

- Les discriminations envers les femmes ont diminué. Le taux d'activité des femmes est passé de $39,3 \%$ en 1970 à $47,9 \%$ en 1994 , contre $59,8 \%$ en moyenne dans l'OCDE. L'écart entre les salaires féminins et masculins s'est réduit à $40,5 \%$ en 1995 , il reste tout de même deux fois plus élevé qu'en France où l'écart est de $20 \%$ en moyenne ${ }^{2}$.

- Un salaire minimum a été institué en 1988 pour les entreprises occupant 10 salariés ou plus, même si son niveau reste faible: il représentait $29 \%$ du salaire moyen des industries manufacturières en 1989, $24 \%$ en 1994, alors que dans les pays de l'OCDE son niveau est généralement compris entre 34 et $56 \%$ du salaire moyen ${ }^{3}$.

- Dans le domaine de l'emploi, la législation du travail n'a pas été significativement modifiée pour ce qui concerne les licenciements. La loi autorise une entreprise à licencier un salarié pour un motif valable moyennant un préavis d'un mois ou le paiement de trente jours de salaire, ce que le salarié peut contester devant une Commission des relations professionnelles. Mais, jusqu'en 1997, la loi n'autorisait pas les licenciements économiques dans un délai rapide, comme dans les autres pays de l'OCDE. De plus, la modification des rapports de forces entre employeurs et syndicats a permis à ces derniers de faire inscrire, dans à peu près un tiers des conventions collectives, une clause stipulant que l'entreprise doit obtenir l'accord du syndicat pour procéder à des licenciements économiques. Ce frein doit être relativisé dans la mesure où, en 1990 , seuls $17,4 \%$ des salariés étaient couverts par des conventions collectives, pour l'essentiel dans les grandes entreprises (Chaebols); à la même date, seuls $10 \%$ des entreprises de moins de 99 salariés avaient signé une convention collective soit avec un syndicat ( $3 \%$ des cas), soit avec un conseil regroupant employeurs et employés ( $7 \%$ des cas) comme le prévoit la loi; $90 \%$ des petites entreprises n'avaient signé aucune convention collective ${ }^{4}$, or les entreprises de moins de 300 salariés

1. Source : KLI, 1993, op. cit. et OCDE, 1996 a, op. cit.

2. Source : Les chiffres concernant la durée du travail et l'écart entre salaires féminin et masculin sont tirés du Rapport annuel des statistiques du travail, BIT, op. cit.

3. OCDE, 1996 a, op. cit.

4. Y. B. Park (1993), Labor in Korea, Séoul, Korea Labor Institute, p. 94-95. 
représentent, en 1994, 71,5\% de l'emploi total. On ajoutera qu'en 1996 $47 \%$ des salariés coréens ont un contrat temporaire, voire sont des tra-

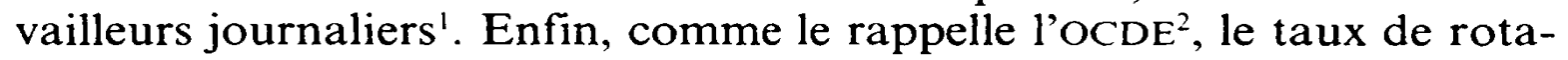
tion annuel du travail mesuré par les départs volontaires étant resté très élevé, de l'ordre de $40 \%$ par an dans le secteur manufacturier en 1993 , une entreprise peut fortement réduire ses effectifs en s'abstenant seulement de pourvoir les emplois vacants.

$\mathrm{Au}$ total, le bilan doit être nuancé. Si l'effervescence sociale qui caractérise la Corée au tournant des années 1980-1990 a mis fin aux excès de la flexibilité quantitative qui existaient auparavant, il n'en demeure pas moins que les entreprises coréennes disposent encore de marges de manœuvre considérables par rapport à leurs concurrentes des pays de l'OCDE. Seuls les Chaebols se plaignaient de ne pouvoir ajuster leurs effectifs aux variations de la production, ce qui explique pourquoi, en décembre 1996, une révision de la législation du travail cherchait à répondre à leur demande au nom de l'alignement sur les normes en vigueur dans les pays de l'OCDE. Le seul changement d'importance concerne les hausses de salaires qui se sont accélérées depuis 1987, dépassant nettement le rythme de progression de la productivité du travail. Quel en a été l'impact sur la compétitivité?

\section{L'évolution de la compétitivité}

Il est d'usage d'analyser l'évolution de la compétitivité-coût avec un indice de coûts unitaires relatifs du travail et la compétitivité-prix avec un indice de prix relatifs à l'exportation. En Corée, les coûts unitaires du travail, après avoir baissé de 16,7\% entre 1980 et 1986, ont augmenté d'environ $66 \%$ entre 1987 et 1991 , mais seulement de $7,4 \%$ entre 1992 et $1996^{3}$. Sous le coup de cette dégradation de la compétitivité-coût, la compétitivité-prix a baissé de 8,7\% entre 1987 et 1991, mais elle s'est améliorée de $12 \%$ entre 1992 et 1996, car les entreprises coréennes ont fait le choix de réduire la profitabilité à l'exportation de $18 \%$. Peut-on en déduire que la flambée des coûts salariaux à partir de 1987 aurait conduit la Corée à une situation critique du point de vue de la compétitivité?

Ce serait oublier le niveau initial très faible des salaires coréens. Une comparaison de la compétitivité-coût en niveau, et non plus en évolution, montre que les coûts unitaires en travail de la Corée restent encore

1. Source: National Statistical Office. Séoul, 13 novembre 1997.

2. Op. cit. p. 114.

3. Ces chiffres ainsi que ceux concernant les prix relatifs à l'exportation ont été calculés d'après les statistiques présentées dans Perspectives économiques de l'OCDE, juin 1997. 
nettement inférieurs à ceux de ses principaux partenaires commerciaux. En 1993 les coûts salariaux unitaires coréens, évalués au taux de change de parité des pouvoirs d'achat, étaient encore de moitié inférieurs à ceux des États-Unis, alors que ceux de Taiwan n'étaient plus que de $20 \%$ inférieurs ${ }^{1}$. Ce faible niveau des salaires permettait de combler le retard de la productivité qui ne représentait que $30 \%$ de celle des États-Unis. On comprend pourquoi le secteur exportateur est capable de supporter à la fois une dégradation de sa compétitivité-coût et une amélioration de sa compétitivité-prix. Mais ces chiffres indiquent qu'à l'avenir, la Corée devra asseoir sa compétitivité sur d'autres bases que le seul coût salarial unitaire. Ceci est d'autant plus vrai que la compétitivité dépend de plus en plus de critères hors coût : qualité des produits, délai de livraison, service après-vente, qui relèvent non pas essentiellement de la flexibilité quantitative, mais surtout de la flexibilité fonctionnelle. Le vrai problème est de savoir comment promouvoir la flexibilité fonctionnelle, tout en préservant la flexibilité quantitative? C'est ce que nous allons voir avec l'exemple de la branche automobile de Hyundai.

\section{Le développement problématique de la flexibilité fonctionnelle}

Hyundai Motor Company (HMC), la filiale automobile de l'un des principaux Chaebols, est représentative des difficultés à promouvoir la flexibilité fonctionnelle dans une entreprise qui a longtemps été le symbole du "paternalisme autoritaire ". HMC se développe particulièrement dans les années 1980 grâce à l'expansion du marché coréen et, surtout, des exportations massives vers les États-Unis d'un modèle d'entrée de gamme dont le principal argument de vente est le faible prix. Cette stratégie, très "fordiste", recherche avant tout la croissance des volumes, nécessaires à l'obtention d'économies d'échelle, et s'appuie sur une organisation du travail de type "paramilitaire" et des relations sociales de type hire and fire, pour imposer faibles salaires, durée et intensité élevées du travail ${ }^{2}$. Telles sont les bases, au niveau micro-économique, de la compétitivité-coût. Dès la fin des années 1970, HMC cherche cependant à développer certaines des innovations organisationnelles japonaises telles que le contrôle de qualité

1. Ces chiffres concernent l'industrie manufacturière. Source: F. Benaroya. D. Janci (1996), La sousévaluation de la monnaie asiatique, CEP1I, Économie internationale, n⿳6, 66, $2^{\mathrm{r}}$ trim., p. 29.

2. Toutes les informations présentées ici s'appuient sur le travail de M. K. Chung (1995), in Search of a Work Position. The Trajectory of Hyundai, $3^{e}$ Rencontre internationale du GERPISA, Les nouveaux modèles industriels, Ministère de l'Enseignement supérieur et de la recherche, Paris, 15-17 juin, mimeo. 
totale, les activités de Kaisen ${ }^{1}$, à travers le développement de cercles de qualité et d'un programme de suggestions des salariés, afin d'améliorer la productivité et la qualité des produits. Mais cette tentative est vouée à l'échec, car elle est totalement incompatible avec le climat social qui résulte du "paternalisme autoritaire». Les ouvriers refusent d'adhérer à ces innovations organisationnelles. C'est pourquoi la priorité est accordée à l'automation pour atteindre les objectifs visés sans modifier l'organisation du travail et les conditions d'emploi. Ce choix se transforme en handicap à la fin des années 1980. A partir de 1987, HMC se trouve confrontée à deux problèmes simultanés. La crise sociale qui se généralise dans toute la Corée à cette époque sera particulièrement intense à $H M C$, qui devra reconnaître l'existence d'un syndicat indépendant, négocier des hausses de salaires, ainsi que des réductions du temps et du rythme de travail. En conséquence, HMC perd rapidement des parts de marché aux États-Unis, d'autant plus que le taux de change du Won s'apprécie vis-à-vis du dollar à partir de 1986. La perte de l'avantage de prix sur le marché américain (qui était évalué à $1110 \$$ en 1986 , pour des modèles comparables) met en valeur la mauvaise qualité des produits et la longueur des délais de livraison, révélant à quel point la compétitivité reposait sur des bases fragiles. C'est pourquoi HMC tentera de relancer les innovations organisationnelles qui participent à la flexibilité fonctionnelle dans le but de trouver des solutions à la crise sociale qui contribuent aussi à améliorer la productivité et la qualité des produits : introduction d'un nouveau système de classification visant à créer des filières de promotion, comme la création du poste de "chef de groupe", encouragement à la rotation sur les postes de travail dans le but de développer la polyvalence des salariés, relance des cercles de qualité et des programmes de suggestions, programme de formation visant à la fois l'amélioration des connaissances et l'esprit coopératif. Ces innovations organisationnelles ont aussi pour objectif de tirer le meilleur parti des équipements en automation programmable introduits en grand nombre dans l'espoir d'améliorer la productivité et la qualité, mais aussi de réduire les effectifs.

Ces initiatives ont rencontré un succès mitigé. Les salariés contestent la désignation des chefs de groupe et voudraient les élire, ce que refuse la direction. La rotation sur les postes de travail, pratiquée par $35 \%$ des ouvriers (selon une enquête réalisée par le syndicat), est le plus souvent organisée au sein du collectif de travail par les salariés eux-mêmes en fonction de la pénibilité de chaque poste et non pas pour se former et

1. Kaisen : améliorations continues, voir glossaire p. 459. 
acquérir des compétences nouvelles. Les salariés considèrent avec méfiance la polyvalence car ils craignent qu'elle soit le moyen d'augmenter l'intensité du travail. De même préfèrent-ils se former au contact d'ouvriers plus qualifiés que dans les centres de formation professionnelle de l'entreprise. Enfin, en cas de problèmes avec les systèmes automatisés, les ouvriers de production stoppent systématiquement la ligne de production et s'en remettent aux services d'entretien.

Cet exemple montre que le développement de la flexibilité fonctionnelle est bien plus ardu, car il touche au cœur même du rapport salarial. Durant la période d'industrialisation rapide, au moment où il s'agissait de mettre en place la production de masse, c'est-à-dire d'accroître les volumes sans souci de la qualité, en combinant mécanisation et paternalisme autoritaire, les gains de productivité étaient élevés et le processus de rattrapage (catching up) était rapide. Aujourd'hui, les étapes suivantes du rattrapage sont plus difficiles à franchir, car la productivité dépend plus étroitement de l'implication des salariés qui est elle-même déterminée par les conditions sociales prévalant au sein des entreprises, et d'une façon plus générale dans la société coréenne. D'autant plus que tous les pays industrialisés sont engagés dans le même processus depuis plusieurs années. Dans ces pays, mais aussi au Brésil et au Mexique, la pression du chômage et l'aggravation des inégalités sociales pèsent d'un poids considérable dans l'implication des salariés et dans la détermination des contreparties qu'ils en obtiennent. Conserver son emploi est souvent considéré comme une contrepartie suffisante. En Corée, au moins jusqu'en 1996, dans un contexte caractérisé par le plein-emploi et un mouvement syndical plus fort que dans certains pays plus riches, le coût des contreparties que devaient accorder les grandes entreprises était proportionnellement plus élevé, d'autant plus que le salaire indirect est peu socialisé par l'État. Quelles seront les conséquences prévisibles de la crise de 1997?

CONCLUSION

La crise financière de l'été 1997 conduit à une restructuration des secteurs bancaires et industriels. Les banques devront cesser d'être de simples guichets accordant des crédits aux Chaebols, qui vont devoir justifier la rentabilité de leurs projets d'investissements, d'autant plus que l'ouverture de l'économie coréenne exigée par le FMI et l'OCDE renforce la concurrence. Les restructurations entraînent à court terme des milliers de licenciements et une augmentation rapide du chômage 
qui devrait atteindre $6 \%$ en 1998. La question qui demeure est de savoir jusqu'à quel point la crise sera l'occasion d'une réforme structurelle de l'économie coréenne assurant la primauté aux mécanismes de marché?

Bien que contraints de réduire leur endettement ${ }^{1}$ et leur rythme d'expansion, les Chaebols constituent plus que jamais l'instrument privilégié d'insertion dans l'économie mondialisée. La diversification, si elle est rendue plus cohérente sur le plan industriel et transparente sur le plan financier, peut devenir un outil efficace de synergie technologique et de diversification des risques.

La crise et l'ouverture économique exercent aussi une pression dans le sens d'une plus grande flexibilité quantitative. Les licenciements économiques pour raison d'urgence, à l'origine de la grève générale de janvier 1997, ainsi que de nouvelles facilités en matière de flexibilité horaire devraient être adoptés en 1998 et conduire à une baisse du coût du travail. La discipline du marché va renforcer son influence au sein des entreprises et on peut en attendre une baisse du coût des contreparties sociales incitant au développement de la flexibilité fonctionnelle. Mais il est douteux que les salariés coréens, qui pensaient au mois de janvier 1997 pouvoir enfin bénéficier des fruits de plusieurs décennies d'effort, se résignent à un retour en arrière durable.

Ces considérations indiquent que la transformation d'un régime d'accumulation régulé par l'État en un régime d'accumulation régulé par le marché sur le mode anglo-saxon est loin d'être acquise. 